\section{(C) OPEN ACCESS}

\title{
Gastric microbial community profiling reveals a dysbiotic cancer-associated microbiota
}

\author{
Rui M Ferreira, ${ }^{1,2}$ Joana Pereira-Marques, ${ }^{1,2,3}$ Ines Pinto-Ribeiro, ${ }^{1,2,4}$ Jose L Costa, 1,2,4 \\ Fatima Carneiro, ${ }^{1,2,4,5}$ Jose C Machado, 1,2,4 Ceu Figueiredo 1,2,4
}

\begin{abstract}
- Additional material is published online only. To view please visit the journal online (http://dx.doi.org/10.1136/ gutjnl-2017-314205).

'i3S - Instituto de Investigação e Inovação em Saúde, Universidade do Porto, Porto, Portugal

Ipatimup - Institute of Molecular Pathology and Immunology of the University of Porto, Porto, Portugal ${ }^{3}$ Institute of Biomedical Sciences Abel Salazar (ICBAS), University of Porto, Porto, Portugal ${ }^{4}$ Faculty of Medicine, University of Porto, Porto, Portugal ${ }^{5}$ Department of Pathology, Centro Hospitalar São João, Porto, Portugal
\end{abstract}

Correspondence to Professor Ceu Figueiredo, Ipatimup - Institute of Molecular Pathology and Immunology of the University of Porto, Rua Júlio Amaral de Carvalho 45, 4200-135 Porto, Portugal; cfigueiredo@ipatimup.pt

Received 24 March 2017 Revised 25 October 2017 Accepted 26 October 2017 Published Online First 4 November 2017

\section{ABSTRACT}

Objective Gastric carcinoma development is triggered by Helicobacter pylori. Chronic H. pylori infection leads to reduced acid secretion, which may allow the growth of a different gastric bacterial community. This change in the microbiome may increase aggression to the gastric mucosa and contribute to malignancy. Our aim was to evaluate the composition of the gastric microbiota in chronic gastritis and in gastric carcinoma.

Design The gastric microbiota was retrospectively investigated in 54 patients with gastric carcinoma and 81 patients with chronic gastritis by 16S rRNA gene profiling, using next-generation sequencing. Differences in microbial composition of the two patient groups were assessed using linear discriminant analysis effect size. Associations between the most relevant taxa and clinical diagnosis were validated by real-time quantitative PCR. Predictive functional profiling of microbial communities was obtained with PICRUSt.

Results The gastric carcinoma microbiota was characterised by reduced microbial diversity, by decreased abundance of Helicobacter and by the enrichment of other bacterial genera, mostly represented by intestinal commensals. The combination of these taxa into a microbial dysbiosis index revealed that dysbiosis has excellent capacity to discriminate between gastritis and gastric carcinoma. Analysis of the functional features of the microbiota was compatible with the presence of a nitrosating microbial community in carcinoma. The major observations were confirmed in validation cohorts from different geographic origins.

Conclusions Detailed analysis of the gastric microbiota revealed for the first time that patients with gastric carcinoma exhibit a dysbiotic microbial community with genotoxic potential, which is distinct from that of patients with chronic gastritis.

\section{INTRODUCTION}

Gastric carcinoma is a major health problem worldwide, with an estimated 1 million new cases every year. ${ }^{1}$ Helicobacter pylori infection plays a crucial role in the initial steps of carcinogenesis by causing enhanced inflammation and progres sive degradation of the architecture and function of the gastric epithelium. ${ }^{2}{ }^{3}$ From a certain point on, however, gastric carcinoma devel opment may be $H$. pylori independent, since colonisation decreases (and is eventually lost) in later steps of carcinogenesis. ${ }^{4}$ Additionally, H. pylori eradication studies have shown that successful eradication

\section{Significance of this study}

What is already known on this subject?

- Helicobacter pylori infection increases the risk for gastric carcinoma by causing chronic inflammation and decreasing the number of acid-producing glands.

- Gastric acid reduction by acid-suppressive drugs results in bacterial overgrowth and high levels of gastric nitrite and $N$-nitrosamine.

- The context of a complex microbiota accelerates the onset and promotes neoplasia in the $H$. pylori insulin-gastrin mouse model of gastric cancer.

What are the new findings?

- The gastric microbiota profile of patients with carcinoma is significantly different from that of patients with chronic gastritis.

- The gastric carcinoma microbiota is dysbiotic and characterised by reduced microbial diversity, reduced Helicobacter abundance and over-representation of bacterial genera that include intestinal commensals.

- The microbial community found in gastric carcinoma has increased nitrosating functions consistent with increased genotoxic potential.

How might it impact on clinical practice in the foreseeable future?

Our results provide a new interpretative frame for understanding the microbial dysbiosis associated with gastric carcinoma, and suggest that alterations in the gastric microbiota may need to be considered to maximise efficacy of preventive and therapeutic strategies tailored at reducing the incidence of gastric carcinoma.

does not completely prevent gastric carcinoma development. ${ }^{5-7}$ These observations suggest that factors other than $H$. pylori contribute to persistent inflammation of the gastric mucosa and to gastric cancer development.

It has been proposed that changes that occur in the stomach as a result of chronic $H$. pylori infec tion leading to decreased acid secretion allow the successful establishment of a new microbiota that contributes to malignant transformation through maintenance of inflammation and conversion of nitrates into $N$-nitrosamines. ${ }^{2}{ }^{3}$ This is supported by earlier studies showing that reduction of gastric 
acid by different types of drugs results in significant intragastric bacterial overgrowth, increased counts of nitrate-reducing bacteria and increased nitrite and $\mathrm{N}$-nitrosamine levels. ${ }^{89}$ This is further supported by studies in the hypergastrinaemic insulin-gastrin (INS-GAS) transgenic mouse model, which showed that $H$. pylori-induced gastric cancer is promoted by the pres ence of a complex gastric microbiota, as these animals develop more tumours than germ-free mice infected with $H$. pylori only. ${ }^{10} 11$

So far, only a very small number of studies characterised the human gastric microbiota in health and disease. Their major findings were that $H$. pylori-negative subjects contained a diverse microbiota in their stomach, whereas in $H$. pylori-infected patients the gastric mucosa was dominated by this species. ${ }^{12-15}$ In the context of gastric carcinogenesis, few studies have been conducted and no particular component of the microbiota has been identified as implicated in gastric carcinoma. ${ }^{15} 16$ The limitations of these studies are the limitations in sensitivity and coverage compared with more recently developed techniques, and, most importantly, the inclusion of very limited numbers of subjects, making it difficult to generate statistically significant conclusions. Therefore, we performed high-throughput profiling of the gastric bacterial communities present in 135 gastric carcinoma cases and chronic gastritis controls, by next-generation sequencing (NGS) of the $16 \mathrm{~S}$ rRNA gene. We used validation cohorts from multiple geographic locations to confirm our findings.

\section{MATERIALS AND METHODS Patients}

Eighty-one individuals with chronic gastritis and 54 with gastric carcinoma were included in the Portuguese discovery cohort (see online supplementary table S1). These were part of a case-control study aimed at investigating risk modifiers for gastric cancer. ${ }^{17} 18$ Subjects with chronic gastritis (mean age 43.6 \pm 7.0 years; male-to-female ratio 39.5:1) were recruited during a screening programme for premalignant lesions of the gastric mucosa and underwent standard gastroscopy at Centro Hospitalar São João (CHSJ). Eleven patients presented glan dular atrophy with foci of intestinal metaplasia. Of these, $1 \mathrm{had}$ mild corpus and moderate antral atrophy and the remaining 10 cases did not have corpus atrophy and had mild $(n=6)$, moderate $(n=2)$ or marked $(n=2)$ atrophy in the antrum (including incisura). Only individuals without evidence of past or present peptic ulcer disease were included. In addition, patients under proton pump inhibitor or antimicrobial treatments were excluded. Patients with gastric carcinoma (mean age 58.8 \pm 13.2 years; male-to-female ratio $1.5: 1$ ) were diagnosed and underwent cancer resection at CHSJ. A validation cohort of an additional 38 gastric specimens from 15 patients with chronic gastritis and 23 patients with gastric carcinoma, diagnosed between 2014 and 2016, were retrieved from the tissue and tumour bank at CHSJ (see online supplementary table S1). All procedures were in accordance with the institutional ethical standards. Samples were delinked and unidenti fied from their donors.

Two additional validation series, consisting of NGS data of the $16 \mathrm{~S}$ rRNA gene of 79 gastric carcinoma cases from a population from China and 53 gastric carcinoma cases from a population from Mexico, were retrieved from the Sequence Read Archive (BioProject PRJNA310127; see online supplementary table S2). ${ }^{19}$

\section{S rRNA gene sequencing}

DNA was isolated from gastric biopsies or surgical specimens of non-neo plastic gastric mucosa adjacent to the tumour, as previously described. ${ }^{17}$ The $16 \mathrm{~S}$ rRNA gene was amplified using primers U789F 5'-TAGATACCCTGGTAGTCC-3' and U1053R 5'-CTGACGACAGCCATGC-3' targeting the V5-V6 hypervariable regions and sequenced in an Ion PGM Torrent platform following manufacturer's instructions. Primers were designed following recommendations reported by Andersson et al, and were extensively analysed using PrimerProspector (see online supplementary figure S1). ${ }^{20} 21$

\section{Sequencing data analysis}

The performance of the UPARSE pipeline was evaluated and compared with that reported for samples of the Human Microbiome Project (HMP) data set. ${ }^{22}$ Using UPARSE (usearch v7.0.1090 i86linux64), reads were filtered by imposing a maximum number of expected errors of 0.5 and a global trimming at 250 nucleotides. ${ }^{22}$ Reads were dereplicated and singletons were discarded. Filtered reads were clustered into operational taxonomic units (OTU) assuming 97\% similarity. Chimeric reads were reference removed using Uchime. ${ }^{22}$ Each OTU was taxonomically assigned using Uclust considering a minimum percentage of similarity to a reference database (Greengenes Named Isolate database, release August 2013) match of $90 \% .^{23}$ Diversity analyses were performed using QIIME (V.1.9). ${ }^{24}$ Alpha diversity was determined by the Shannon index and with Good's estimator of coverage. Differences in alpha diversity were assessed by the t-test controlled with $10^{3}$ Monte Carlo permutations. Beta diversity was assessed by unweighted and weighted UniFrac distance matrices and visualised by principal coordinate analysis (PCoA), controlled by $10^{3}$ jackknife replicates. ${ }^{25}$ Sample clustering in beta diversity analysis was tested using analysis of similarity (ANOSIM) with $10^{4}$ bootstrap replications. ${ }^{26}$ Comparisons between distance matrices were evaluated by the Mantel correlation controlled with $10^{4}$ permutations.

\section{Taxonomic discovery analysis}

Statistically significant differences in the relative abundance of taxa associated with groups of patients were performed using linear discriminant analysis (LDA) effect size (LEfSe). ${ }^{27}$ Only taxa with LDA greater than 4 at a $P$ value $<0.05$ were considered significantly enriched.

\section{Real-time quantitative PCR}

Sequencing results were confirmed by quantitative PCR (qPCR) (see online supplementary table S3).

\section{Functional metagenome predictions}

For functional metagenome prediction, we captured OTU representative sequences from Greengenes database using the USEARCH global alignment command and discarding reads that did not hit the reference database. Reconstruction of the metagenome was performed using PICRUSt. ${ }^{28}$ Accuracy of the predicted metagenomes was assessed by determining the nearest sequenced taxon index. Predicted functional genes were categorised into Clusters of Orthologous Groups (COG) and into Kyoto Encyclopedia of Genes and Genome (KEGG) orthology (KO), and compared across patient groups using STAMP. ${ }^{29}$ Statistical differences in COG and KO frequencies were determined by White's non-parametric t-test with a Benjamini-Hochberg false discovery rate correction to adjust $\mathrm{P}$ values for multiple testing. ${ }^{30}$ 
For further details, see online supplementary information.

\section{RESULTS}

\section{Quality control of 16 S rRNA microbiota profiling}

In the present study, we compared the gastric microbiota of patients with chronic gastritis with that of patients with gastric carcinoma by NGS of the $16 \mathrm{~S}$ rRNA gene. After sequencing and quality filtering, more than 10.8 million reads were obtained corresponding to a mean of 80261 reads and 178 OTUs per sample (see online supplementary figure S2). On average, patients with chronic gastritis had a significantly higher number of reads (86 957) than patients with cancer (67 954; $P<0.05)$. However, the number of OTUs was not significantly different between the two patient groups (186 and 169 OTUs, respectively; $P=0.071$ ). To control for the number of false OTUs and to measure the number of biologically meaningful OTUs, we classified them according to similarity shared with sequences of the Greengenes Named Isolated database (see online supplementary figure S2). In our data set, the frequencies of misleading and valid OTUs were similar to those reported for the HMP data set processed with the UPARSE pipeline. ${ }^{22}$

To assure consistency between amplification and sequencing sets, 32 randomly selected samples were used to test reproducibility. The intraclass correlation coefficients showed good reproducibility for the assessment of the Shannon index, for the UniFrac distances and for the relative abundance of phyla (see online supplementary table S4). In conclusion, our approach provides the most in-depth characterisation of the gastric microbiota so far and generates robust and consistent data.

\section{The gastric microbiota profile differs in chronic gastritis and gastric carcinoma}

To evaluate alterations in the microbiota structure between patients with chronic gastritis and gastric carcinoma, we measured microbial alpha diversity (ie, within sample diversity) and beta diversity (ie, diversity between samples). By measuring alpha diversity using the Shannon index, we found that patients with gastric carcinoma had significantly decreased microbial diversity in compar ison with patients with chronic gastritis (figure $1 \mathrm{~A}, P=0.003$; online supplementary figure S3). To ensure good estimation of bacterial diversity, we measured the proportion of total bacterial species represented in samples of each patient group by the Good's estimator of coverage. Estimated coverage ranged from 0.94 to 0.98 in chronic gastritis and from 0.95 to 0.99 in gastric carcinoma $(P=0.225)$, suggesting that the $16 \mathrm{~S}$ rRNA results from each (chronic gastritis and gastric carcinoma) library represent the majority of bacteria present in the gastric mucosa (figure 1B).

Beta diversity was calculated using both unweighted (ie, qualitative) and weighted (ie, quantitative) UniFrac phylogenetic distance matrices, and visualised in PCoA plots. The total
A

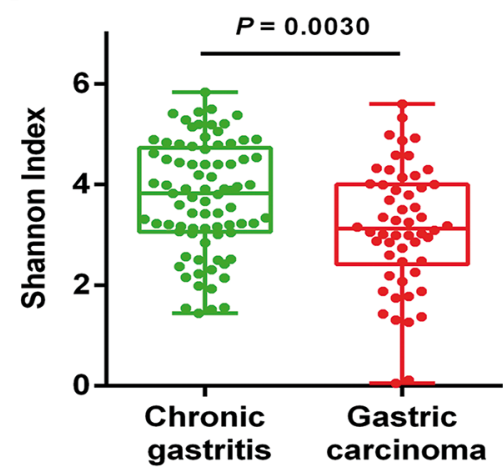

C

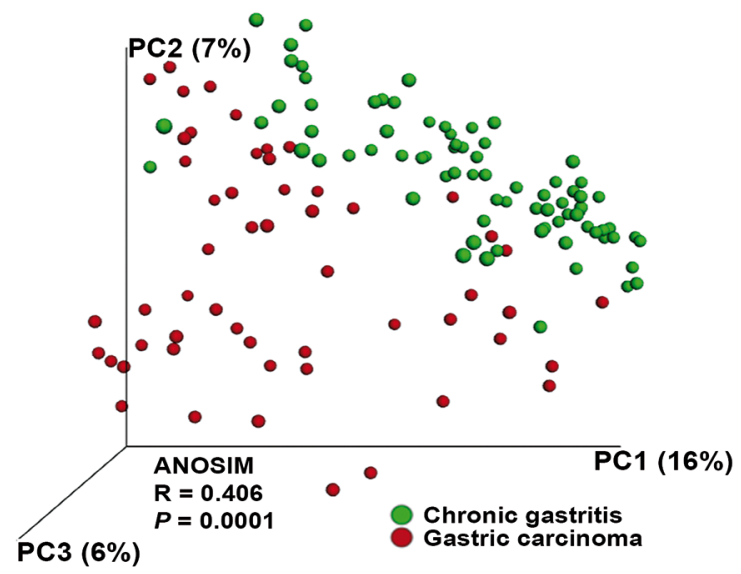

B

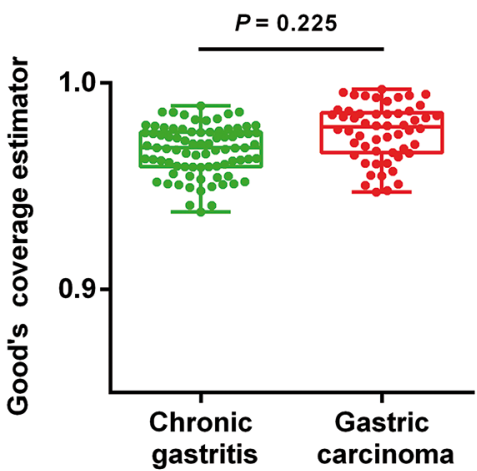

D

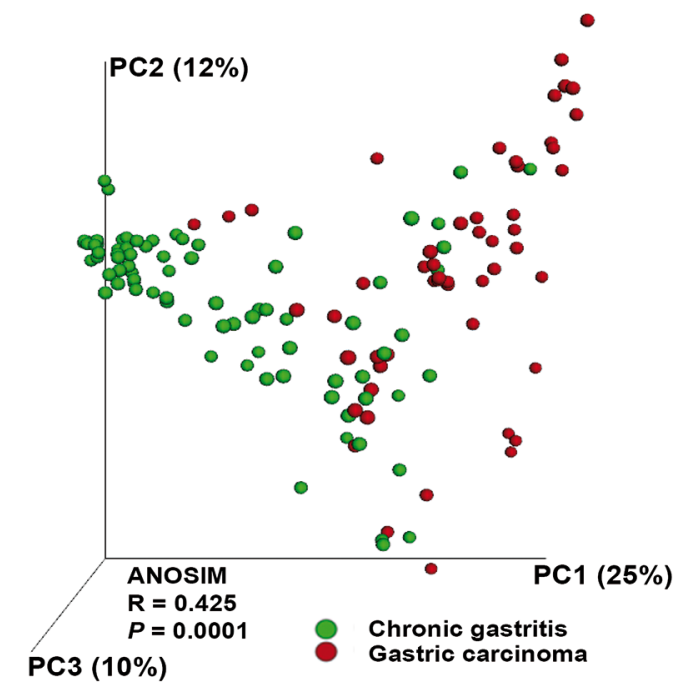

Figure 1 The gastric microbiota profile differs in chronic gastritis and gastric carcinoma. (A) Shannon index of diversity in patients with chronic gastritis and gastric carcinoma. (B) Good's estimator of coverage, measuring the proportion of total bacterial species represented in samples of each group of patients. Principal coordinate analysis ( $\mathrm{PCOA})$ plots of $(\mathrm{C})$ unweighted and (D) weighted UniFrac distances in which samples were coloured by clinical outcome. The percentage of diversity captured by each coordinate is shown. ANOSIM, analysis of similarity. 
diversity captured by the top three principal coordinates was $29 \%$ and $47 \%$ for unweighted and weighted UniFrac, respectively. The microbiota composition of patients with gastric carcinoma was significantly different from that of patients with chronic gastritis (ANOSIM R $=0.406, P=0.0001$; and $\mathrm{R}=0.425$, $P=0.0001$, for unweighted and weighted distances, respectively; figure $1 \mathrm{C}, \mathrm{D})$.

Since age is an established risk factor for gastric carcinoma, and since patients with carcinoma were significantly older than patients with gastritis in our series (see online supplementary table S1), we next addressed whether the microbial profile was different between younger and older patients. Overall, increasing age could differentiate the microbiota profiles of the full sample set (see online supplementary figure S4A,B). However, when we performed age-matched comparisons of the microbiota in patients with chronic gastritis and carcinoma, we observed statistically significant differences in the unweighted and weighted UniFrac distances (see online supplementary figure S4C,D), reinforcing that the microbiota composition is different in the two clinical settings. Also in the age-matched comparisons, significantly decreased microbial alpha diversity was found in patients with gastric carcinoma (see online supplementary figure S4E, $P=0.0096$ ).
No statistically significant differences in the microbiota profiles of gastric carcinoma cases were observed for gender, histological type and tumour location (see online supplementary figure S5).

In patients with chronic gastritis, we could not detect differences in the alpha diversity and beta diversity between patients with non-atrophic gastritis $(n=70)$ and patients with glandular atrophy $(n=11$; online supplementary figure $S 6)$, and therefore they were pooled together for the analyses.

These results show that there is a significant reduction in microbial diversity in gastric carcinoma. Furthermore, the fact that the weighted UniFrac captured more diversity than unweighted metrics suggests that alterations in the relative abundance of taxa are a major contributor for microbiota differences between gastritis and gastric carcinoma.

\section{The influence of $\boldsymbol{H}$. pylori in the microbiota composition of chronic gastritis and gastric carcinoma}

Overall, the gastric microbiota was dominated by five phyla: Proteobacteria (69.3\%), Firmicutes (14.7\%), Bacteroidetes (9.0\%), Actinobacteria (4.3\%) and Fusobacteria (1.3\%). Although these phyla were present in the two patient groups in the same order of relative abundance, the gastric carcinoma microbiota
A

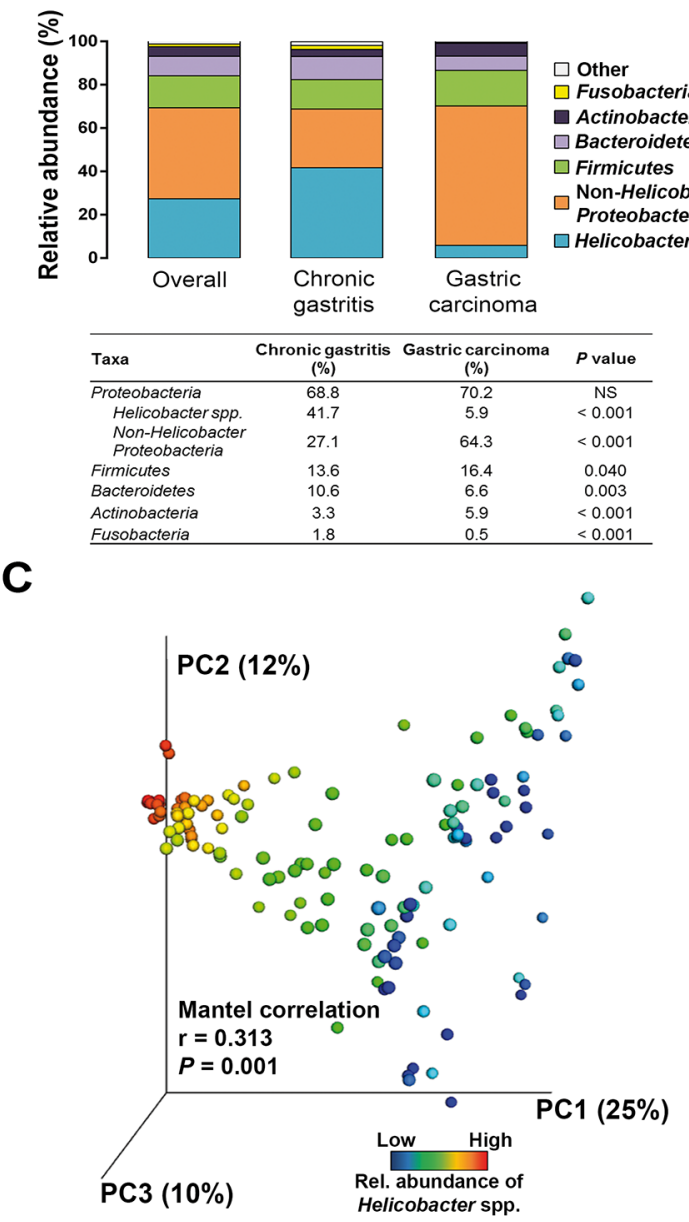

B

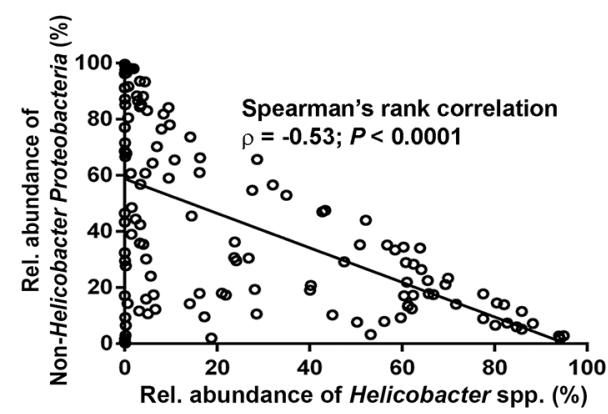

D

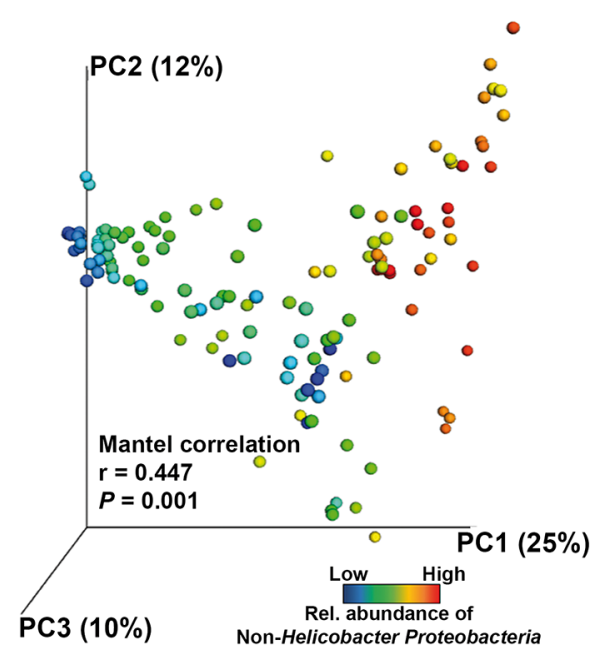

Figure 2 The influence of Helicobacter pylori in the microbiota composition of chronic gastritis and gastric carcinoma. (A) Relative abundance of phyla in all subjects and in each group of patients. (B) Spearman's rank correlation between relative abundance of Helicobacter spp. and nonHelicobacter Proteobacteria in all patients. Principal coordinate analysis (PCOA) plots of the weighted UniFrac distance matrix coloured by (C) increasing relative abundance of Helicobacter and of (D) non-Helicobacter Proteobacteria. 
had an over-representation of Actinobacteria $(\mathrm{P}<0.001)$ and Firmicutes $(\mathrm{P}=0.040)$, and lower abundance of Bacteroidetes $(P=0.003)$ and Fusobacteria $(P<0.001$; figure $2 \mathrm{~A})$.

When reads assigned to Proteobacteria into Helicobacter spp. and non-Helicobacter Proteobacteria were separated, a significant reduction in the abundance of Helicobacter $(P<0.001)$ and an over-representation of non-Helicobacter Proteobacteria were detected in gastric carcinoma $(P<0.001$; figure $2 \mathrm{~A})$. Accordingly, a significant negative correlation was found between these taxa $(\mathrm{r}=-0.53, P<0.0001$; figure $2 \mathrm{~B})$. In support of the above, the microbiota profile of the two patient groups could be distinguished by the abundance of Helicobacter (Mantel correlation, $\mathrm{r}=0.313, P=0.001$; figure $2 \mathrm{C}$ ) and by the abundance of non-Helicobacter Proteobacteria (Mantel correlation, $r=0.447$, $P=0.001$; figure 2D).

Regarding Helicobacter spp. in chronic gastritis, the mean relative abundance of this genus was $41.7 \%$, but varied considerably between patients from $0.01 \%$ to $94.9 \%$ (figure $2 \mathrm{~A}$ ). The relative abundance of Helicobacter was inversely correlated with the abundance of non-Helicobacter Proteobacteria $(\mathrm{r}=-0.59$, $P<0.0001)$, Firmicutes $(\mathrm{r}=-0.49, \quad P<0.0001)$, Bacteroidetes $(\mathrm{r}=-0.43, P<0.0001)$ and Actinobacteria $(\mathrm{r}=-0.54$, $P<0.0001$; online supplementary table S5). In contrast, the great majority of patients with gastric carcinoma (80\%) had a relative abundance of Helicobacter below 5\%, including eight patients in which Helicobacter reads were not detected by NGS. The abundance of Helicobacter in gastric carcinoma was correlated with that of Bacteroidetes and Fusobacteria (see online supplementary table S5).

Overall, these results show that for high taxonomic levels the stomach microbial communities differ in chronic gastritis and gastric carcinoma, suggesting that major changes also occur at lower taxonomic levels. Additionally, our data validate that Helicobacter exists in the gastric carcinoma microbiota as a low abundant or absent genus.

\section{Specific microbial taxa are associated with gastric carcinoma}

To identify the most relevant taxa responsible for the differences between clinical diagnoses, we conducted LEfSe analysis. ${ }^{27}$ This analysis identified 29 taxa, including 10 genera, which were differentially abundant in the two patient groups (figure 3A,B). In gastric carcinoma, an enrichment in Proteobacteria taxa was observed, including the genera Phyllobacterium and Achromobacter and the families Xanthomonadaceae and Enterobacteriaceae. Although no specific genus could be identified within the Xanthomonadaceae, in the Enterobacteriaceae, the genus Citrobacter was identified as being significantly enriched in gastric carcinoma. Additionally, Lactobacillus, Clostridium and Rhodococcus were also significantly more abundant in gastric carcinoma. Helicobacter, Neisseria, Prevotella and Streptococcus were most abundant in the microbiota of patients with chronic gastritis. Results of the LEfSe analysis in the age-matched subset closely recapitulated the bacteria taxa differentially abundant in the two patient groups (see online supplementary figure S4F).

To show that relationships among disease-associated taxa did not depend on differences observed in the abundance of Helicobacter, we conducted a reanalysis subtracting the Helicobacter reads from the data set. Considering the same parameters in the LEfSe analysis, we confirmed the enrichment of Streptococcus, Prevotella and Neisseria in chronic gastritis (see online supplementary figure S7). Additionally, we identified an enrichment in two Proteobacteria taxa, Novosphingobium and Pasteurellales, and in two Bacteroidetes families, Chitinophagaceae and
Saprospirae. In gastric carcinoma, no additional taxa were detected after removing the Helicobacter reads from the data set.

To validate gastric carcinoma-enriched and depleted taxa, we used NGS data from an independent Chinese cohort of 79 gastric carcinoma cases. In this data set, and in agreement with the results obtained in the Portuguese discovery cohort, we could detect statistically significant enrichment in Citrobacter, Rhodococcus, Lactobacillus and Phyllobacterium, and depletion in Helicobacter and Neisseria (figure 3C). Clostridium reads were enriched in gastric carcinoma cases from the Chinese population, although not reaching statistical significance in the LEfSe analysis. Achromobacter reads were not detected in the Chinese validation cohort.

To demonstrate that our data were not biased by the microbiota profiling pipeline used, LEfSe results were validated by qPCR in the Portuguese discovery cohort using both genus-specific and universal primers. We confirmed significant decreases in the abundance of Helicobacter and Neisseria, and significant increases of Achromobacter, Citrobacter, Phyllobacterium, Clostridium, Rhodococcus and Lactobacillus in gastric carcinoma in comparison with chronic gastritis (figure 3D). We have additionally used a second validation cohort from Portugal, and with the exception of Prevotella and Streptococcus, we were able to confirm the alterations in the abundance of the eight genera as identified by the original LEfSe analysis (figure 3E).

Next, we compared gastric carcinoma cases and chronic gastritis control subjects for the prevalence of specific taxa. As shown in table 1 , the six genera significantly enriched in gastric carcinoma and identified by LEfSe analysis were also significantly more prevalent in patients with gastric carcinoma than in patients with chronic gastritis. In logistic regression models with carriage of the genera Phyllobacterium, Achromobacter, Citrobacter, Lactobacillus, Clostridium or Rhodococcus as the independent variables, and gastric carcinoma as the dependent variable, the ORs for gastric carcinoma were 3.5 (95\% CI 1.7 to 7), 20.5 (95\% CI 7.4 to 59), 9.9 (95\% CI 4.3 to 23), 6.3 (95\% CI 2.9 to 14 ), 5.7 (95\% CI 2.2 to 15 ) and 4.2 (95\% CI 1.7 to 11 ), respectively. The associations remained significant after adjustment for age and sex.

\section{Microbial dysbiosis is associated with gastric carcinoma}

We next combined the 10 most relevant taxa that characterised each group of patients and calculated the microbial dysbiosis index (MDI). ${ }^{31}$ The gastric microbiota of patients with gastric carcinoma had a higher MDI than that of patients with chronic gastritis both in the discovery cohort and in the validation cohorts $(P<0.0001$; figure 4A). Similar findings were observed in the age-matched subset of the discovery cohort (see online supplementary figure $54 G$ ). Likewise, significantly higher MDI was observed in the microbiota of patients with gastric carcinoma in comparison with that of patients with chronic gastritis, as assessed using $\mathrm{qPCR}$ in the Portuguese validation cohort $(P<0.0001$; figure 4B). The MDI showed an inverse correlation with the alpha diversity $(\mathrm{r}=-0.262, P=0.005$; figure $4 \mathrm{C})$ and a direct correlation with the beta diversity $(r=0.208, P=0.001$; figure 4D), resulting in a clear differentiation gradient among samples. These results demonstrate that the gastric carcinoma microbiota has a high degree of dysbiosis, consistent with reduced bacterial diversity.

We also evaluated whether the MDI could be used to discriminate between chronic gastritis and gastric carcinoma. In receiver operating characteristics (ROC) analysis, the MDI showed excellent performance in identifying gastric 
A

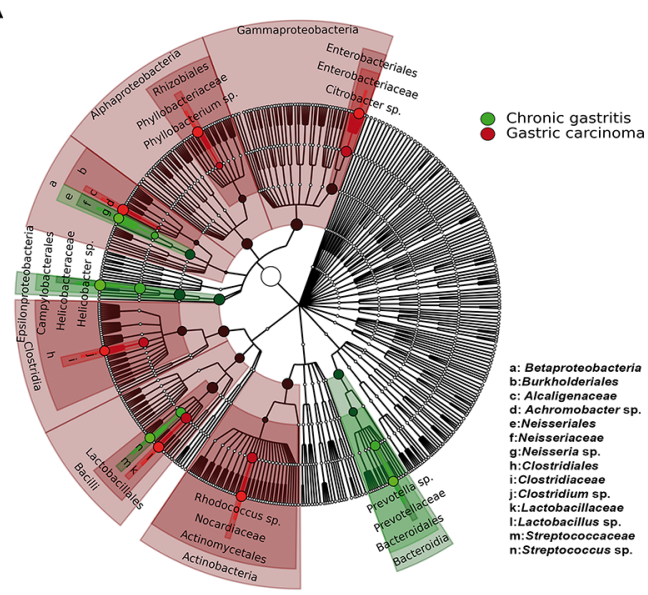

C

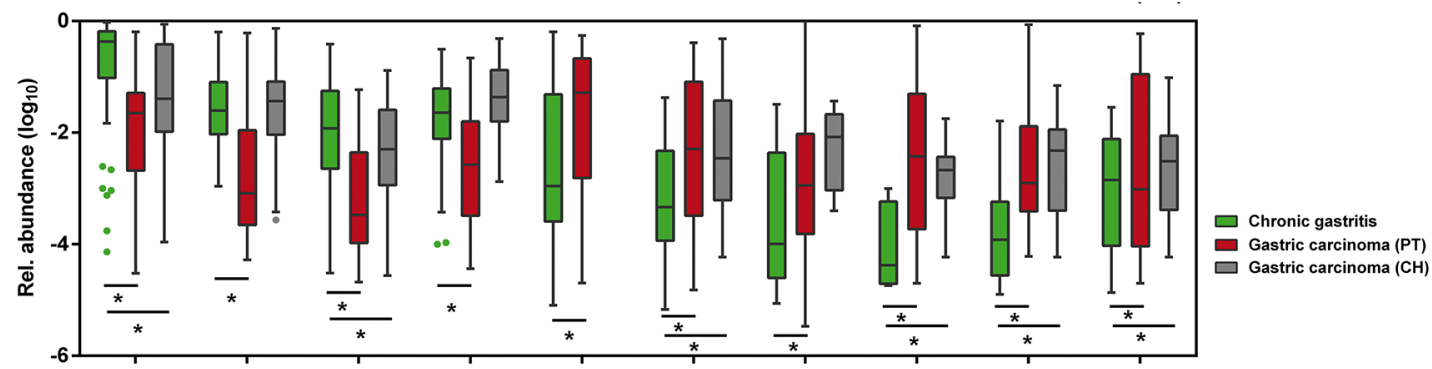

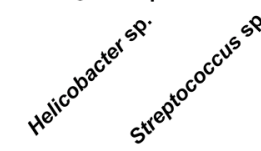

D

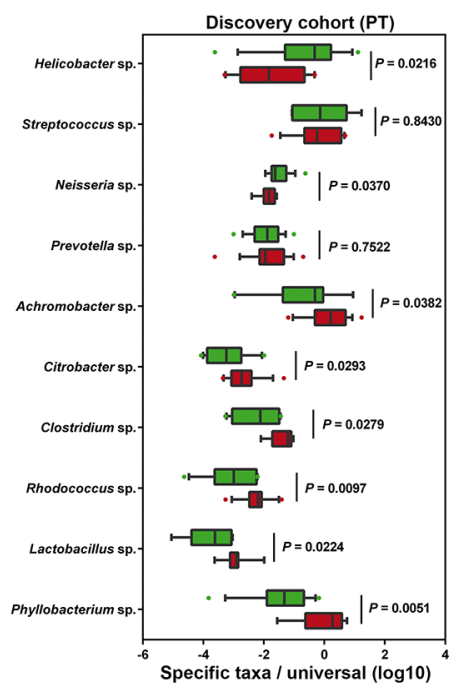

B

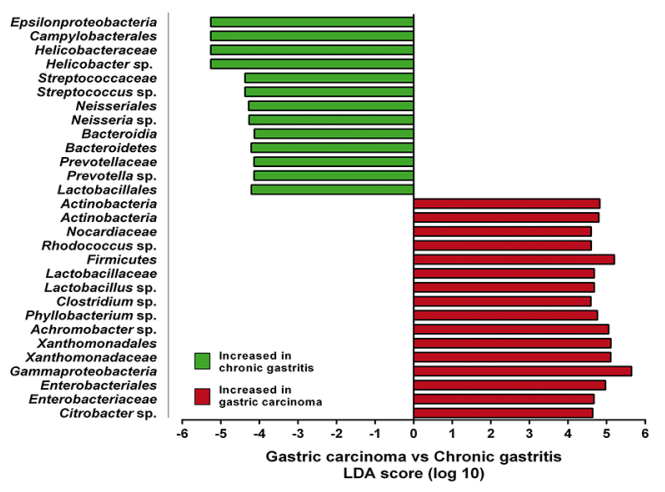
differential abundance of Prevotella and Streptococcus, we recalculated the MDI excluding these genera. This analysis confirmed higher levels of dysbiosis in the gastric carcinoma microbiota in all cohorts, and similar AUCs in the ROC analysis (see online supplementary figure S9). figure S8). 


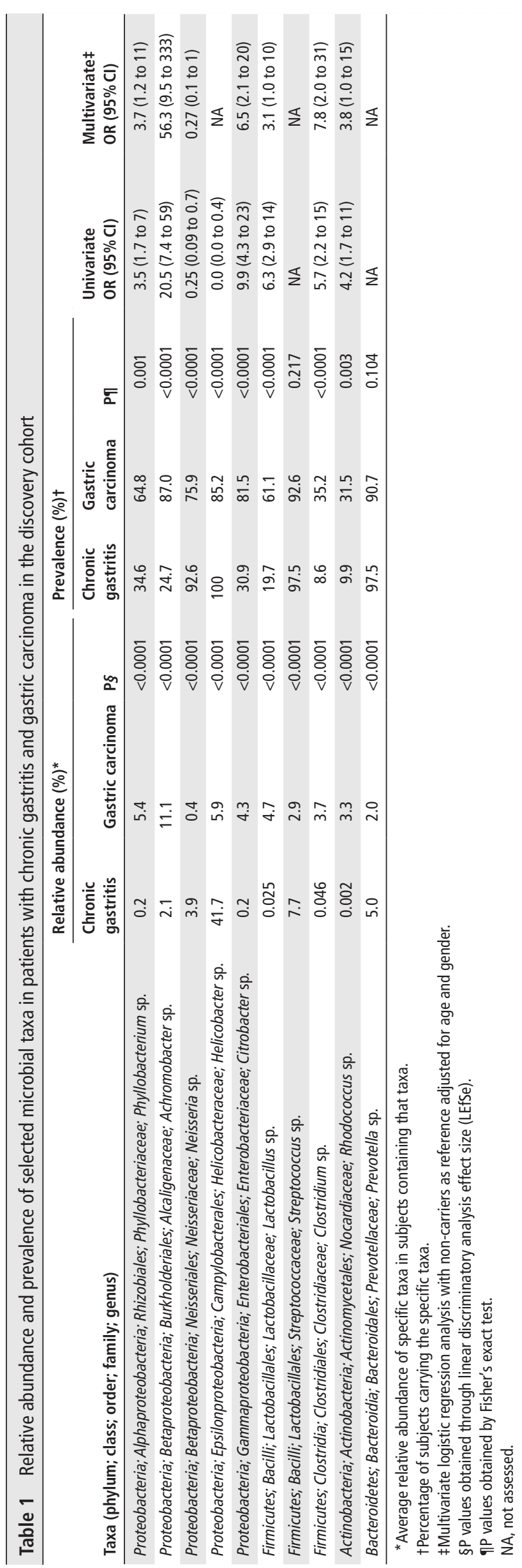

\section{The gastric carcinoma microbiota is characterised by nitrosating bacteria}

To infer the metagenome functional content based on the microbial community profiles obtained from the 16S rRNA gene sequences we used PICRUSt. ${ }^{28}$ Overall, the microbial communities present in patients with gastric carcinoma and chronic gastritis could be distinguished based on their functions (see online supplementary figure S10A). The predicted KEGG pathways significantly enriched in gastric carcinoma included membrane transport, carbohydrate metabolism, transcription, xenobiotics biodegradation and metabolism, cellular processes and signalling, metabolism, signal transduction, amino acid metabolism and lipid metabolism (see online supplementary table S6).

Because it has been hypothesised that nitrate-reducing bacterial species contribute to gastric malignant transformation by increasing intragastric concentrations of nitrite and $\mathrm{N}$-nitroso compounds, we next compared chronic gastritis and gastric carcinoma regarding the microbial functional features involved in those metabolic reactions (see online supplementary table S7). The full reconstitution of the metagenomes showed that the functional composition of the total gastric carcinoma microbiota had increased nitrate reductase functions, which promote the reduction of nitrate to nitrite, and nitrite reductase functions, which promote the reduction of nitrite to nitric oxide, when compared with that of the chronic gastritis (figure 5A,B). Similar results were obtained when the 10 genera differentially abundant in the two patient groups were analysed (see online supplementary figure S10B,E). Collectively, these data provide evidence that a microbial community with genotoxic potential is present in gastric carcinoma.

\section{DISCUSSION}

We have profiled the gastric microbiota associated with chronic gastritis and gastric carcinoma in the largest and most in-depth study to date. We have demonstrated that the gastric microbiota composition in patients with gastric carcinoma is significantly different from that of patients with chronic gastritis. Gastric carcinoma dysbiosis was consistent with a microbial community with genotoxic potential, characterised by reduced microbial diversity, reduced Helicobacter abundance and over-representation of new bacterial genera. The major findings revealed in the Portuguese discovery cohort were confirmed in additional validation cohorts from multiple geographic locations.

In our study, the gastric microbial communities in gastritis and carcinoma were structurally different, with decreased alpha diversity in carcinoma. Our findings are supported by previous data pointing to lower bacterial diversity among five patients with gastric cancer compared with five patients with non-atrophic gastritis. ${ }^{15}$ Also supporting our data, and while our paper was in revision, another paper was published in Gut that identified significant decreases in microbial richness in intestinal metaplasia and in gastric carcinoma compared with superficial gastritis. ${ }^{32}$ Reduced microbial diversity has now been recognised as a feature of disease states, including inflammatory diseases and cancer. ${ }^{31} 3334$ For example, patients with colorectal cancer had decreased overall microbial community diversity in comparison to healthy controls. ${ }^{34}$

In terms of the composition of the gastric microbiota, Proteobacteria, Firmicutes, Bacteroidetes, Actinobacteria and Fusobacteria were the five dominant phyla in the stomach, in accordance with previous descriptions. ${ }^{12} 1420$ At the phylum level, we have already identified differences between the two patient groups, 
A

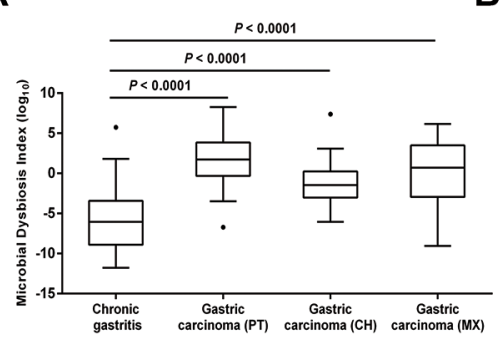

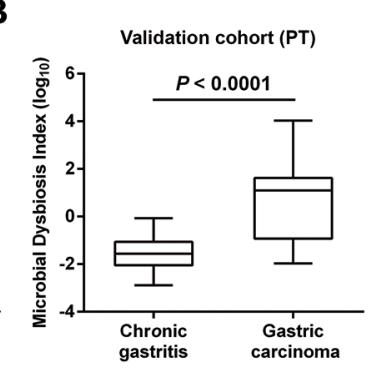

C

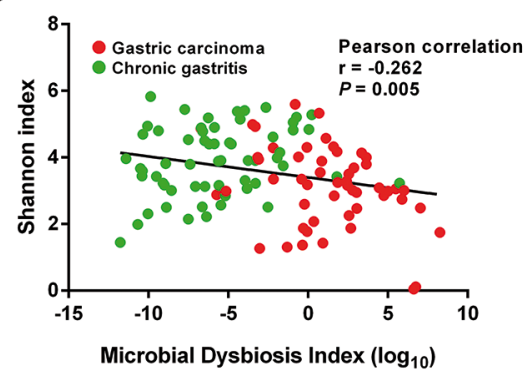

D

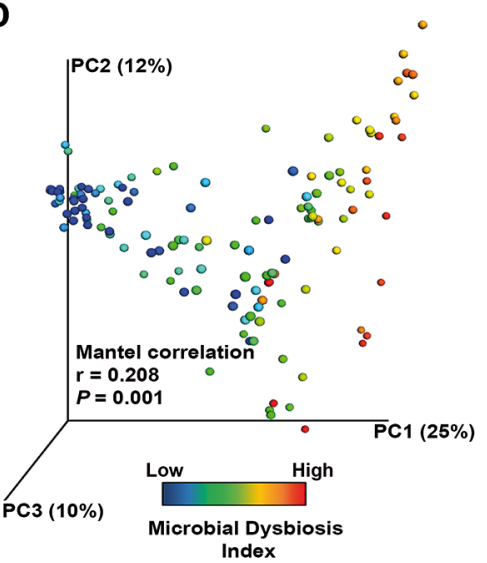

E

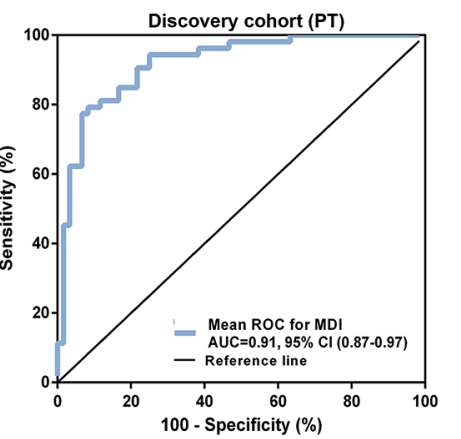

$\mathbf{F}$

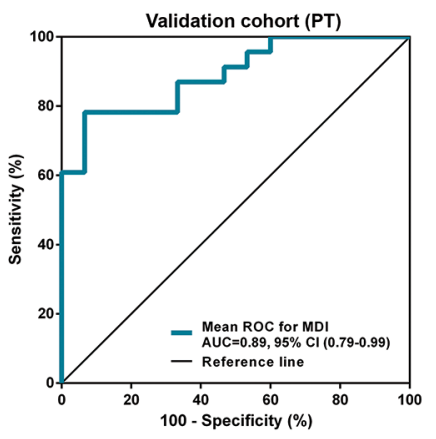

Figure 4 Microbial dysbiosis is associated with gastric carcinoma. (A) Box plot showing the MDI in the discovery cohort and in the Chinese and Mexican validation cohorts. Significance was obtained by one-way analysis of variance (ANOVA) corrected with Holm-Sidak test for multiple comparisons. (B) Box plot showing the MDI of the Portuguese validation cohort. Significance was obtained by Student's t-test. (C) Negative Pearson's correlation between MDI and Shannon index. (D) Principal coordinate analysis (PCOA) plot of the weighted Unifrac distance coloured by increasing MDI. The percentage of diversity captured by each coordinate is shown. Mantel correlations controlled with $10^{4}$ permutations were used to compare distances. (E,F) ROC curves analysis to evaluate the discriminatory potential of MDI in gastric carcinoma detection in the discovery cohort (E) and in the Portuguese validation cohort (F). AUC, area under the curve; MDI, microbial dysbiosis index; ROC, receiver operating characteristic.

with increased abundance of non-Helicobacter Proteobacteria, Firmicutes and Actinobacteria in cancer specimens. Importantly, by applying the LEfSe algorithm that was validated for high-dimensional microbiome data sets, we were able to determine the bacterial taxa that most likely explain differences between clinical diagnoses. ${ }^{27}$ Additionally, in this study, the major taxonomic differences that were detected after analyses of sequencing-generated and bioinformatics-treated data were further validated by real-time qPCR assays.

In chronic gastritis, and as expected, Helicobacter was detected as the most abundant genus. Streptococcus, Prevotella and Neisseria were also found significantly overabundant in this patient group, although Streptococcus and Prevotella could not be confirmed by qPCR. Nevertheless, these genera have been identified earlier in $\mathrm{H}$. pylori-positive and negative gastritis by $16 \mathrm{~S}$ rDNA and rRNA sequencing, and by culture from gastric juice and gastric biopsies. ${ }^{12} 143536$ In fact, they are among the five most commonly found genera in the non-neoplastic stomach. ${ }^{12}{ }^{20}{ }^{37}$ Streptococcus, Prevotella and Neisseria are commensals of the oral cavity and oesophagus and whether they constitute transient or active resident stomach microbes is not yet clarified. Interestingly, in a study that compared the gastric microbiota compositions in $\mathrm{H}$. pylori-positive individuals from two populations with high and low gastric cancer risks in Colombia, Neisseria and Streptococcus were among the genera that occurred more abundantly in individuals from the low gastric cancer risk region. ${ }^{38}$
In gastric carcinoma, there was a significant decrease in $\mathrm{Heli}$ cobacter abundance, and several taxa were found to be significantly more abundant. These included Citrobacter, Clostridium, Lactobacillus, Achromobacter and Rhodococcus, which reside in the intestinal mucosa as commensals but can be opportunistic pathogens. ${ }^{39} 40$ Phyllobacterium, which are environmental bacteria commonly found in plant roots, were too identified at higher abundance in gastric carcinoma ${ }^{41}$ All genera significantly overabundant in gastric carcinoma were also significantly more prevalent in gastric carcinoma cases than in chronic gastritis control patients, and these associations remained significant after adjustment for age and sex. In line with our results, in a study that combined terminal restriction fragment length polymorphism with $16 \mathrm{~S}$ rRNA gene cloning and sequencing, Lactobacillus was one of the dominating genera in 10 Swedish patients with gastric cancer. ${ }^{16}$ Additionally, the use of the microarray G3 PhyloChip to characterise the stomach microbiota of Mexican patients revealed a trend towards the increase of a Lactobacillus sp from non-atrophic gastritis, to intestinal metaplasia, to gastric cancer. ${ }^{15}$ Moreover, Citrobacter, Clostridium and Lactobacillus have all been cultured from the gastric juice of achlorhydric patients, patients undergoing acid suppression therapy and patients with gastric cancer. ${ }^{42-44}$ Interestingly, infection with Citrobacter rodentium species increases epithelial cell proliferation and promotes colonic tumour formation in genetically susceptible mice as well as in chemically initiated colon carcinogenesis. ${ }^{45} 46$ 
A

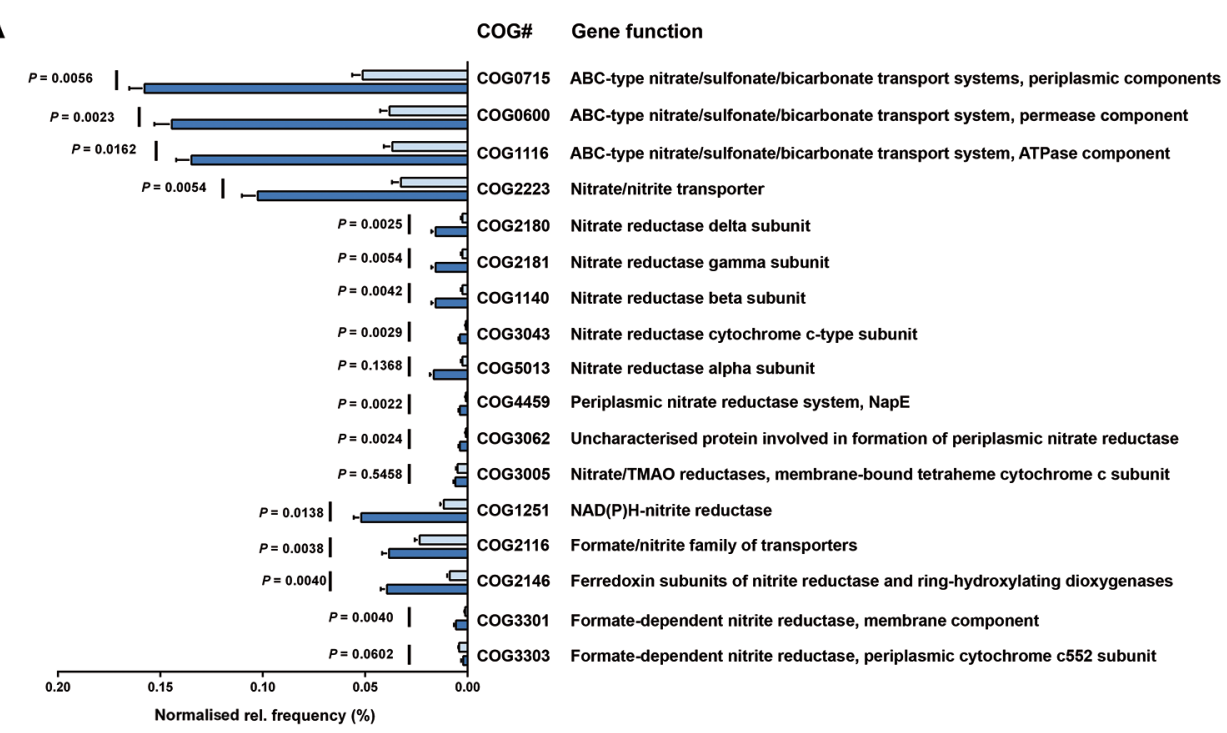

Nitrate reductase

Nitrite reductase

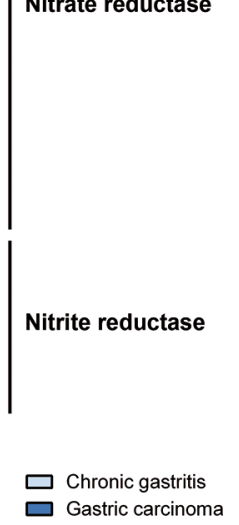

B

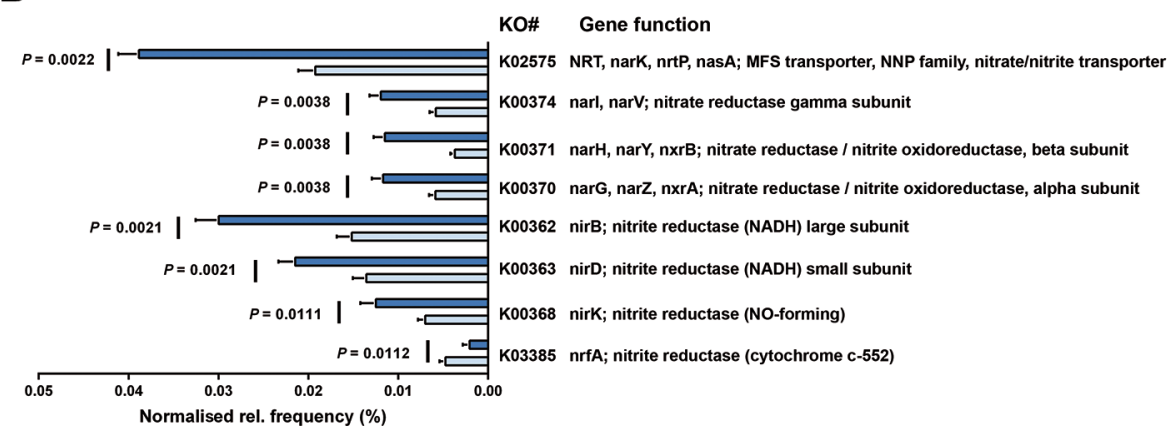

Nitrate reductase

Nitrite reductase

Figure 5 The gastric carcinoma microbiota is characterised by nitrosating bacteria. Functional classification of the predicted metagenome content of the microbiota of chronic gastritis and gastric carcinoma using (A) COG and (B) KO. The normalised relative frequency of nitrate reductase and nitrite reductase in patients with chronic gastritis and gastric carcinoma is shown. Significance was considered for adjusted $P<0.05$. COG, Clusters of Orthologous Groups; KO, Kyoto Encyclopedia of Genes and Genome (KEGG) orthology; NADH, nicotinamide adenine dinucleotide; NO, nitric oxide; TMAO, trimethylamine $\mathrm{N}$-oxide.

The integration of data from the most relevant genera that characterised each patient group allowed us to calculate the dysbiosis index that showed excellent capacity to discriminate between gastritis and gastric carcinoma. Furthermore, the dysbiosis index had improved sensitivity and specificity to detect gastric carcinoma in comparison with the use of single genera, which suggests that changes in the microbial community rather than individual taxa contribute to gastric carcinoma development.

After having analysed the diversity and composition of the gastric microbiota and the microbial features associated with gastric carcinoma, we addressed the functional features of the microbiota. Specifically, we demonstrated that in comparison with chronic gastritis, the gastric carcinoma microbiota has increased nitrate reductase and nitrite reductase functions. This observation is compatible with the hypothesis that during carcinogenesis, changes in the stomach mucosa that lead to decreased acid secretion allow the growth of bacteria that are able to reduce nitrate to nitrite, a precursor of carcinogenic $\mathrm{N}$-nitroso compounds. ${ }^{2}$

Taken together our results and previously published data, we propose that colonisation with bacteria other than $H$. pylori, namely gut commensals, contributes to alter the equilibrium between the 'resident' gastric microbiota and the host.
This dysbiotic microbial community, by sustaining the gastric inflammatory process, and through its intrinsic genotoxic potential, may augment the risk for $H$. pylori-related gastric carcinoma development. In line with our proposal, experimental evidence in the INS-GAS model showed that commensal intestinal bacteria play a role in the promotion of gastric cancer. ${ }^{12}{ }^{13}$ Lertpiriyapong et al showed that mice harbouring a complex intestinal microbiota, and mice colonised with a restricted intestinal microbiota (that includes Clostridium and Lactobacillus), had an accelerated onset and progression of gastric cancer secondary to $H$. pylori infection. These mice also developed more severe gastric histopathology and higher expression levels of proinflammatory genes in comparison to germ-free mice (infected or not with $H$. pylori) and mice harbouring a complex or a restricted intestinal microbiota. ${ }^{11}$

Although our study is limited by its retrospective nature, and by the low number of patients with true premalignant lesions, our findings are consistent with a shift in the gastric microbial community structure along gastric carcinogenesis. In this sense, prospective follow-up studies of patients with premalignant lesions, successfully eradicated or not for $H$. pylori infection, would be crucial to ascertain the pathogenic effect of microbial dysbiosis in the progression to carcinoma. Additional studies to address the effect of dysbiosis or of candidate 
bacterial species in an animal model of gastric carcinogenesis can also be considered, and in that regard, a humanised mouse model that better mimics the human immune response could be particularly informative. Ultimately, understanding the microbiota dynamics along gastric carcinogenesis may impact gastric carcinoma prevention and treatment strategies of patients with precancerous disease.

Contributors Study concept and design: CF, JCM. Data acquisition, analysis and interpretation: CF, JCM, RMF, JPM, IPR, JLC, FC. Drafting of the manuscript or revising it critically for important intellectual content: CF, JCM, RMF, JPM, IPR, JLC, FC. Obtained funding: CF, JCM.

Funding This research was supported by a Worldwide Cancer Research grant to CF and JCM (Reference 16-1352). RMF, JPM and IPR have fellowships from Fundação para a Ciência e a Tecnologia (FCT; SFRH/BPD/84084/2012, PD/BD/114014/2015 and SFRH/BD/110803/2015, respectively) through Programa Operacional Capital Humano (POCH) and the European Union. JPM's fellowship is in the framework of FCT's PhD Programme BiotechHealth (Ref PD/0016/2012). i3S-Instituto de Investigação e Inovação em Saúde is funded by Fundo Europeu de Desenvolvimento Regional (FEDER) funds through the COMPETE 2020-Operacional Programme for Competitiveness and Internationalisation (POCI), Portugal 2020, and by Portuguese funds through Fundação para a Ciência e a Tecnologia (FCT)/Ministério da Ciência, Tecnologia e Inovação (POCI-01-0145-FEDER-007274).

\section{Competing interests None declared.}

Patient consent Detail has been removed from this case description/these case descriptions to ensure anonymity. The editors and reviewers have seen the detailed information available and are satisfied that the information backs up the case the authors are making.

Ethics approval Ethics Committee Centro Hospitalar São João.

Provenance and peer review Not commissioned; externally peer reviewed.

Open Access This is an Open Access article distributed in accordance with the terms of the Creative Commons Attribution (CC BY 4.0) license, which permits others to distribute, remix, adapt and build upon this work, for commercial use, provided the original work is properly cited. See: http://creativecommons.org/ licenses/by/4.0/

(c) Article author(s) (or their employer(s) unless otherwise stated in the text of the article) 2018. All rights reserved. No commercial use is permitted unless otherwise expressly granted.

\section{REFERENCES}

1 Ferlay J, Soerjomataram I, Dikshit R, et al. Cancer incidence and mortality worldwide: sources, methods and major patterns in GLOBOCAN 2012. Int J Cancer 2015;136:E359-6.

2 Correa P. Human gastric carcinogenesis: a multistep and multifactorial process-first American cancer society award lecture on cancer epidemiology and prevention. Cancer Res 1992;52:6735-40.

3 Plottel CS, Blaser MJ. Microbiome and malignancy. Cell Host Microbe 2011;10:324-35.

4 El-Omar EM, Oien K, El-Nujumi A, et al. Helicobacter pylori infection and chronic gastric acid hyposecretion. Gastroenterology 1997;113:15-24.

5 Fukase K, Kato M, Kikuchi S, et al. Effect of eradication of Helicobacter pylori on incidence of metachronous gastric carcinoma after endoscopic resection of early gastric cancer: an open-label, randomised controlled trial. Lancet 2008:372:392-7

6 Ma JL, Zhang L, Brown LM, et al. Fifteen-year effects of Helicobacter pylori, garlic, and vitamin treatments on gastric cancer incidence and mortality. J Nat/ Cancer Inst 2012;104:488-92.

7 Wong BC, Lam SK, Wong WM, et al. Helicobacter pylori eradication to prevent gastric cancer in a high-risk region of China: a randomized controlled trial. JAMA 2004;291:187-94.

8 Stockbruegger RW. Bacterial overgrowth as a consequence of reduced gastric acidity. Scand J Gastroenterol Supp/ 1985;111:7-15.

9 Leach SA, Thompson M, Hill M. Bacterially catalysed N-nitrosation reactions and their relative importance in the human stomach. Carcinogenesis 1987;8:1907-12.

10 Lofgren JL, Whary MT, Ge Z, et al. Lack of commensal flora in helicobacter pyloriinfected INS-GAS mice reduces gastritis and delays intraepithelial neoplasia. Gastroenterol 2011;140:210-20.

11 Lertpiriyapong K, Whary MT, Muthupalani S, et al. Gastric colonisation with a restricted commensal microbiota replicates the promotion of neoplastic lesions by diverse intestinal microbiota in the Helicobacter pylori INS-GAS mouse model of gastric carcinogenesis. Gut 2014;63:54-63.

12 Bik EM, Eckburg PB, Gill SR, et al. Molecular analysis of the bacterial microbiota in the human stomach. Proc Natl Acad Sci U S A 2006;103:732-7.
13 Maldonado-Contreras A, Goldfarb KC, Godoy-Vitorino F, et al. Structure of the human gastric bacterial community in relation to Helicobacter pylori status. Isme J 2011:5:574-9.

14 Delgado S, Cabrera-Rubio R, Mira A, et al. Microbiological survey of the human gastric ecosystem using culturing and pyrosequencing methods. Microb Ecol 2013;65:763-72.

15 Aviles-Jimenez F, Vazquez-Jimenez F, Medrano-Guzman R, et al. Stomach microbiota composition varies between patients with non-atrophic gastritis and patients with intestinal type of gastric cancer. Sci Rep 2014;4:4202.

16 Dicksved J, Lindberg M, Rosenquist M, et al. Molecular characterization of the stomach microbiota in patients with gastric cancer and in controls. J Med Microbiol 2009:58:509-16.

17 Figueiredo C, Machado JC, Pharoah P, et al. Helicobacter pylori and interleukin 1 genotyping: an opportunity to identify high-risk individuals for gastric carcinoma. J Natl Cancer Inst 2002;94:1680-7.

18 Machado JC, Figueiredo C, Canedo P, et al. A proinflammatory genetic profile increases the risk for chronic atrophic gastritis and gastric carcinoma. Gastroenterol 2003:125:364-71.

$19 \mathrm{Yu} \mathrm{G}$, Torres J, Hu N, et al. Molecular characterization of the human stomach microbiota in gastric cancer patients. Front Cell Infect Microbiol 2017;7:302.

20 Andersson AF, Lindberg $M$, Jakobsson $\mathrm{H}$, et al. Comparative analysis of human gut microbiota by barcoded pyrosequencing. PLoS One 2008;3:e2836.

21 Walters WA, Caporaso JG, Lauber CL, et al. PrimerProspector: de novo design and taxonomic analysis of barcoded polymerase chain reaction primers. Bioinformatics 2011;27:1159-61.

22 Edgar RC. UPARSE: highly accurate OTU sequences from microbial amplicon reads. Nat Methods 2013;10:996-8.

23 Edgar RC. Search and clustering orders of magnitude faster than BLAST. Bioinformatics 2010;26:2460-1.

24 Caporaso JG, Kuczynski J, Stombaugh J, et al. QIIME allows analysis of highthroughput community sequencing data. Nat Methods 2010;7:335-6.

25 DeSantis TZ, Hugenholtz P, Larsen N, et al. Greengenes, a chimera-checked 16S rRNA gene database and workbench compatible with ARB. App/ Environ Microbiol 2006;72:5069-72.

26 Anderson MJ. A new method for non-parametric multivariate analysis of variance. Austral Ecology 2001;26:32-46.

27 Segata N, Izard J, Waldron L, et al. Metagenomic biomarker discovery and explanation. Genome Biol 2011;12:R60.

28 Langille MG, Zaneveld J, Caporaso JG, et al. Predictive functional profiling of microbial communities using 16S rRNA marker gene sequences. Nat Biotechnol 2013;31:814-21

29 Parks DH, Tyson GW, Hugenholtz P, et al. STAMP: statistical analysis of taxonomic and functional profiles. Bioinformatics 2014;30:3123-4.

30 White JR, Nagarajan N, Pop M. Statistical methods for detecting differentially abundant features in clinical metagenomic samples. PLoS Comput Biol 2009:5:e1000352.

31 Gevers D, Kugathasan S, Denson LA, et al. The treatment-naive microbiome in newonset Crohn's disease. Cell Host Microbe 2014;15:382-92.

32 Coker 00, Dai Z, Nie Y, et al. Mucosal microbiome dysbiosis in gastric carcinogenesis. Gut 2017.doi: 10.1136/gutjnl-2017-314281. [Epub ahead of print 1 Aug 2017].

33 Lepage $\mathrm{P}$, Häsler R, Spehlmann ME, et al. Twin study indicates loss of interaction between microbiota and mucosa of patients with ulcerative colitis. Gastroenterol 2011;141:227-36.

34 Ahn J, Sinha R, Pei Z, et al. Human gut microbiome and risk for colorectal cancer. J Natl Cancer Inst 2013;105:1907-11.

35 Thorens J, Froehlich F, Schwizer W, et al. Bacterial overgrowth during treatment with omeprazole compared with cimetidine: a prospective randomised double blind study. Gut 1996:39:54-9.

36 Schulz C, Schütte K, Koch N, et al. The active bacterial assemblages of the upper GI tract in individuals with and without Helicobacter infection. Gut 2016.doi: 10.1136/ gutjnl-2016-312904. [Epub ahead of print 5 Dec 2016].

37 Li XX, Wong GL, To KF, et al. Bacterial microbiota profiling in gastritis without Helicobacter pylori infection or non-steroidal anti-inflammatory drug use. PLoS One 2009;4:e7985.

38 Yang I, Woltemate S, Piazuelo MB, et al. Different gastric microbiota compositions in two human populations with high and low gastric cancer risk in Colombia. Sci Rep 2016;6:18594

39 Kelly CP, LaMont JT. Clostridium difficile--more difficult than ever. N Eng/ J Med 2008;359:1932-40.

40 Rajilić-Stojanović M, de Vos WM. The first 1000 cultured species of the human gastrointestinal microbiota. FEMS Microbiol Rev 2014;38:996-1047.

41 Jiao YS, Yan H, Ji ZJ, et al. Phyllobacterium sophorae sp. nov., a symbiotic bacterium isolated from root nodules of Sophora flavescens. Int J Syst Evol Microbiol 2015;65:399-406.

42 Forsythe SJ, Dolby JM, Webster AD, et al. Nitrate- and nitrite-reducing bacteria in the achlorhydric stomach. J Med Microbiol 1988;25:253-9. 


\section{Stomach}

43 Sjöstedt S, Heimdahl A, Kager L, et al. Microbial colonization of the oropharynx, esophagus and stomach in patients with gastric diseases. Eur J Clin Microbiol 1985;4:49-51.

44 Mowat C, Williams C, Gillen D, et al. Omeprazole, Helicobacter pylori status, and alterations in the intragastric milieu facilitating bacterial N-nitrosation. Gastroenterol 2000;119:339-47.
45 Barthold SW, Jonas AM. Morphogenesis of early 1, 2-dimethylhydrazine-induced lesions and latent period reduction of colon carcinogenesis in mice by a variant of Citrobacter freundii. Cancer Res 1977;37:4352-60.

46 Newman JV, Kosaka T, Sheppard BJ, et al. Bacterial infection promotes colon tumorigenesis in Apc(Min/+) mice. J Infect Dis 2001;184:227-30. 